\title{
Internet of Everything and Educational Cyber Physical Systems for University 4.0
}

\author{
Samia Bachir and Angel Abenia \\ University of Pau and Pays Adour, Pau, France \\ name.lastname@univ-pau.fr
}

\begin{abstract}
The Internet of Things (IoT) is overwhelming education due to the expansion of smart connected devices. This offers an open gate for knowledge access and ubiquitous learning. However, many challenges remain with regard to educational system policies, at university in particular. A consistent and harmonious platform, that could bring together the different aspects of learning/teaching with the smartness of things, to offer a better learning/educational experience, has not yet been reached. The aim of this paper is to illustrate the different components of the Internet of Everything (IoE) educational ecosystem that should be taken into consideration before generating the learning/teaching processes. We propose the concept of Educational Cyber Physical System as a key element for monitoring the educational environment. We also give some examples illustrating the implementation of such processes.
\end{abstract}

Keywords: Internet of Everything, Educational Ecosystem, Educational Cyber Physical System, University 4.0

\section{Introduction}

The evolution of information technology has revolutionised the way people and objects behave in everyday lives. This is due to the emergence of Internet of Things (IoT) and to the Internet of Everything (IoE), especially in the last decade. This fourth wave, as described in [1], was preceded by three others. The first was categorized by the arrival of computers in the late 1960s where one computer was allocated to several people. Then, a second wave raised after a decade to refer one personal computer to one person. After the appearance of embedded and ubiquitous computing, a third wave occurred to designate many computers to one person.

Different revolutions have taken place in other fields to raise a fourth generation thanks to the Internet of Everything. Industry 4.0 is one of the concerned fields where different research works have started to bring standardisation efforts to create mature contributions in the field. Key elements of Industry 4.0, such as Cyber Physical Systems (CPS), coupled with the Autonomic Computing (AC) principles have lead us to rethink the educational ecosystem of education and thus proposing a fourth generation of University, correspondingly. 
In this paper, we address the following research question: How could a teaching/learning context affect the different interactions between and within the different components of the Internet of Everything educational ecosystem that are managed by the bias of Educational Cyber Physical Systems?. In order to answer this inquiry, we refine the educational ecosystem based on the Internet of Everything paradigm and define its main components, as well as its implementations according to different levels of use throughout the whole educational journey. The remainder of this paper is organized as follow: Section 2 presents the related works covering different elements. Section 3 focuses on a refined educational ecosystem based on the Internet of Everything. A definition of Educational Cyber Physical System is then proposed in section 4. In order to illustrate this concept, proposed scenarios are also explored. Conclusion and future works will resume the paper.

\section{Related works}

University 4.0 has started to gain attention from the educational community. It is still in its infancy and at an earlier stage as an explored research field. For the time being, there are barely any research papers which could be cited or relied on. For this purpose, we build upon ideas from Autonomic Computing (AC) perspective and Industry 4.0 principles to propose a reference framework for the fourth revolution of such educational environment.

$\mathrm{AC}$ is considered as a holistic vision of self-managing capabilities in a system [13. The latters cover different aspects of self-management that implement control loops (monitor, analyse, plan and execute) which collect details from the system and act accordingly, as stated in [6. Control loops are executed by autonomic managers to bring forward significant information to another level of treatment ensured by orchestrating autonomic managers which execute control loops in order to make decisions in a top level of management and orchestrate the different autonomic managers.

Industry 4.0 was first raised among the German industrial community in 2011. It is a complex initiative that embraces several overlapping areas 10. It was defined by Herman et al. [9] as the integration of complex physical machinery and devices with networked sensors and software, used to predict, control and plan for better business and societal outcomes. In such manufacturing environment, smart machines, installations, workpieces and other components exchange data and information in real time which represents a shift from rigid, centralised factory control systems to decentralised intelligence. This revolution is founded on a set of technologies which together promote the fourth revolution, happening currently in our world. Internet of things, Cyber Physical Systems, Cloud Computing, etc. are the basis of Industry 4.0.

On the other hand, IoT has effected the education business model. Several value propositions [2] are empowering, directly or not, students' achievement. One of these value propositions concerns the real-time personalization of learning experiences. This could engage more students [2]. A recent review was pub- 
lished in [16 about exploring IoT in education. Interesting efforts have been conducted to improve the educational ecosystem based on the Internet of Things. We classify them according to the way IoT are used. We opt for the following classification to show precisely the contributions of IoT to improving learning and teaching processes.

- Learning/Teaching of Internet of Things: This classification concerns the teaching and leaning of Internet of Things as a learning subject. The aim is to teach/learn the different core knowledge of the subject. [5], [15, [17, [3] are examples of such research works. Education 4.0 could be classified in this type of IoT exploitation. It focuses on teaching/learning IoT to prepare future professionals who will have the required competences and skills of the subject and then will be able to work on an IoT equipped environment like Industry 4.0.

- Learning/Teaching by Internet of Things: This classification concerns the use of Internet of Things as an artifact to acquire other knowledge. 20, 12 , are examples of research works which focused on such aspect. Experiment based Learning is one of the conducted pedagogies that could be viewed as a way to serve knowledge through the bias of smart objects manipulation.

- Learning/Teaching based on Internet of Things: This classification is not really explored in improving teaching and learning processes. However, it is addressed in some research works to monitor students' healthcare or in classroom access control, like in [19], [14].

- Another category could be drawn according to further uses of IoT in the educational context, which are not directly linked to learning and teaching. These applications focus on energy management, enhancing safety, reducing cost, improving comfort, etc. [2].

According to this classification, we consider research efforts are not explored enough to directly improve educational processes based on IoE. However, they are in an advanced level for the other categories. We thus adopt the positioning of our work on the third categorisation of IoE application (Learning/teaching based on IoT). This does not exclude the possibility of a further association between the other purposes of IoE applications. Our aim is to improve learning and teaching occuring in a connected environment. The collected data from different learning resources or others are monitored, collected and then analysed for a decision-making process to better manage the educational process.

\section{Internet of Everything Ecosystem in Education}

Many definitions have been proposed for Internet of Things within the existant literature. A review about different propositions is provided in [16]. As it was considered, in [12], as an ecosystem that is able to scale and exploit the existing infrastructure of embedded and connected devices, this definition fits well with the expansion that then has occurred with the Internet of Everything (IoE). Silva and Braga [7] argue that IoE is the next evolutionary step of Internet of 


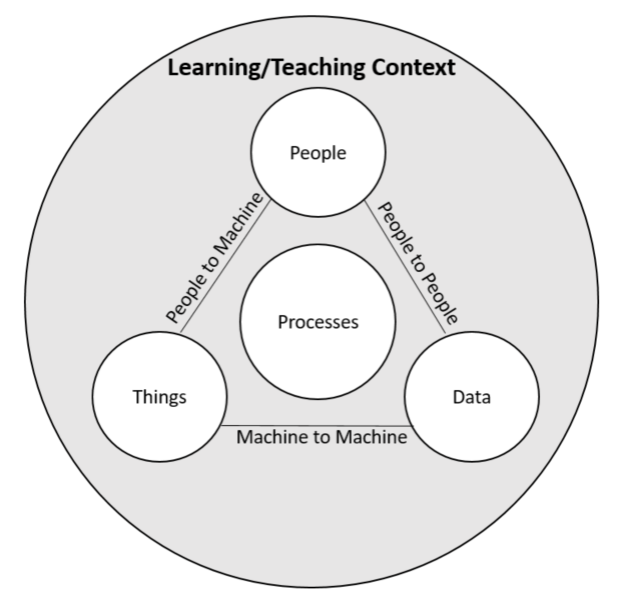

Fig. 1. Main components of the Educational Internet of Everything Ecosystem (revised from [7])

Things where not only embedded devices are making up the network but also people, processes and data have to be taken into account. Few works have been explored in the scientific area of this new paradigm. [7].

As depicted in Fig.1, in the educational ecosystem:

- "People" refers to the students, teachers, administrators or other stakeholders who are involved in the learning/teaching processes.

- "Objects" refers not only to physical devices that can establish connection with the Internet and utilize sensors to capture environment information (as it is presented in [7]), actuators to act on the environment, but also to smart learning resources that can establish connection with the internet for a , directed or in-directed, knowledge access (e.g computer, telepresence-robot).

- "Processes" refers to how people and objects must interact to generate data that can be transformed into usable knowledge. These processes allow information to be addressed to the right people, at the right time and properly [7. Examples will be provided later in the next section.

- "Data" refers to all the different data flows coming from historical information about people, objects, processes and the interactions between the different elements. Cloud computing and learning analytics are examples of technologies for data management.

- Learning/teaching context: We propose to add the concept of learning/teaching context to illustrate a key element of the educational ecosystem. We believe that the general context, where the learning and teaching occur, affects all the different types of interactions between and within the elements listed above. In the literature [18, the context was almost relied basically on the knowledge-to-be-taught and the psychology of learners. However, it is not the case with the arrival of the Internet of Everything and the different designs of physical and virtual spaces. Little details about 


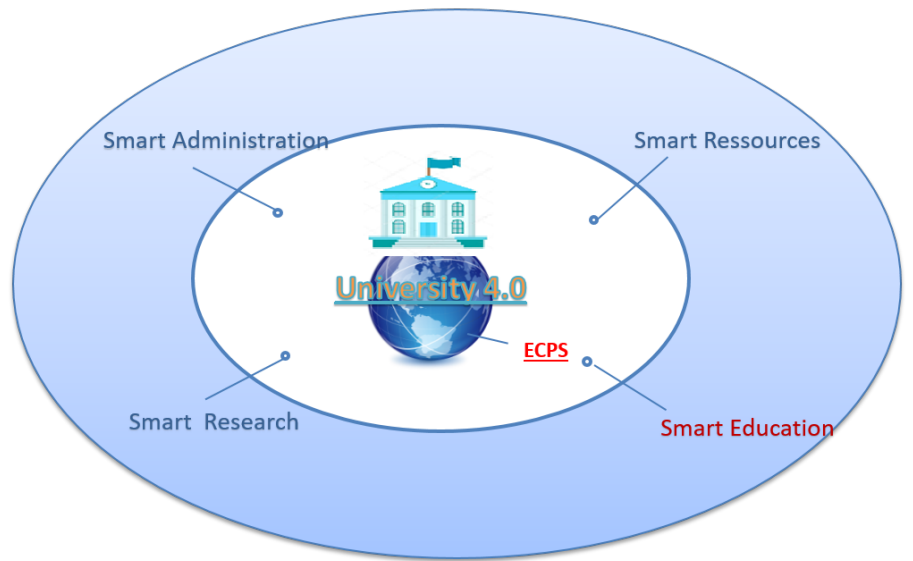

Fig. 2. University 4.0

the context would alter all the predefined processes. We will see later how these elements of context (e.g. Physical space (Auditorium, classroom, eeducation), the number and nature of objects, number of people (present, tele-present), long/medium/short term learning/teaching planning, etc.) are important for Educational Cyber Physical System in generating the required learning/teaching processes.

Putting the different elements of the ecosystem together offers a rich educational experience. At the same time, various nested challenges could be encountered. The interoperability of different computer systems, the establishment of an accurate collaboration scheme between such systems, environments with intelligent software agents to better support the teaching learning process are among the challenges listed in [7]. In the following section, we will explore how such parameters from the learning/teaching context element could affect the scenarisations as well as the interactions between the different elements (people to people, machine to machine, people to machine).

\section{Educational Cyber Physical System for University 4.0 - Proposed Scenarios}

As shown in Fig. 2, there are various aspects within the university as an institutional entity: educational, administrative, research, etc. In our research, we focus on educational aspects and more precisely on improving directly learning and teaching based on a connected environment with everything (people, data, processes, objects, learning/teaching context). However, we present a key concept that could be defined in different levels of use. It is Educational Cyber Physical System (ECPS). To the best of our knowledge, very few studies have focused on the establishment of a definition of ECPS where teaching and learning could occur in both virtual and physical spaces. An attempt was presented in [4] in which explicit representation of the different structural components and 
their correspondent relations or behaviors were not given. Actually, ECPS stands from two complementary directions: Educational purposes, discussed above in the previous section, and CPS.

CPS are systems making the fusion of a real world with a virtual one. According to [11, it consists of two main functional components :

- The advanced connectivity that ensures real-time data acquisition from the physical world and information feedback from the cyber space.

- An intelligent data management, analytics and computational capability that constructs the cyber space.

Hence, based on these elements and IoE ecosystem components as well, we define an ECPS as the contextualisation of the different physical elements of the IoE educational ecosystem (people, objects) to generate the required processes that should be implemented. Data collection and analysis are in the cyber level in order to monitor and supervise the physical world and adapt the processes when it is needed. This monitoring is based on executing control loops (monitor, analyse, plan and execute), induced from the Autonomic Computing perspective.

We consider that the different parameters of the learning/teaching context affect the processes configuration, and so the interactions between and within the different educational elements. We believe that there are three main types of processes which refer to the learning outcomes throughout the whole learning journey (knowledge, competence, expertise [8]):

- Classroom processes: refer to a combination of a set of activities occurring in a physical/virtual classroom. The learning outcomes of such process are knowledge. As presented in [8], knowledge management must address its acquisition, creation, storage, redistribution and application. Learners and teachers are the main actors of this management which highly depends to the choice of pedagogy .

- Course processes: cover the strategy of learning/teaching which highly depends on the knowledge-to-be-taught. Competences are the learning outcomes of this kind of process. They imply the ability to demonstrate a consistent level of performances.

- Curriculum processes: cover the strategy of learning/teaching which highly depends on the learners background/performances/needs. This will lead to expertise acquisition which comprises related value judgements, knowledge and skill sets, lived experiences, and problem solving abilities.

For instance, the definition of temporal parameters (short/medium/long term) and the educational staff roles (teacher / course manager / studies director) allows us to determine the process type we are dealing with (classroom / course / curriculum), respectively. A context parameter can intervene in one or more types of processes. Table 1 shows some examples of context parameters and their interventions in different types of processes. The latters are provided in the following subsections. 
Table 1. The intervention of context parameters in different types of processes

\begin{tabular}{|l|c|c|c|}
\hline & Classroom process & Course process & Curriculum process \\
\hline Process administrator & Teacher & Course manager & Studies director \\
\hline Process duration & short (hours) & medium (weeks) & long (semester/year) \\
\hline Learners prerequisites & - & - & X \\
\hline Pedagogy & $\mathrm{X}$ & $\mathrm{X}$ & - \\
\hline Knowledge-to-be-taught & - & $\mathrm{X}$ & - \\
\hline Course typologic structure & $\mathrm{X}$ & $\mathrm{X}$ & - \\
\hline (Tele-)Presence & $\mathrm{X}$ & - & - \\
\hline Things & $\mathrm{X}$ & - & - \\
\hline
\end{tabular}

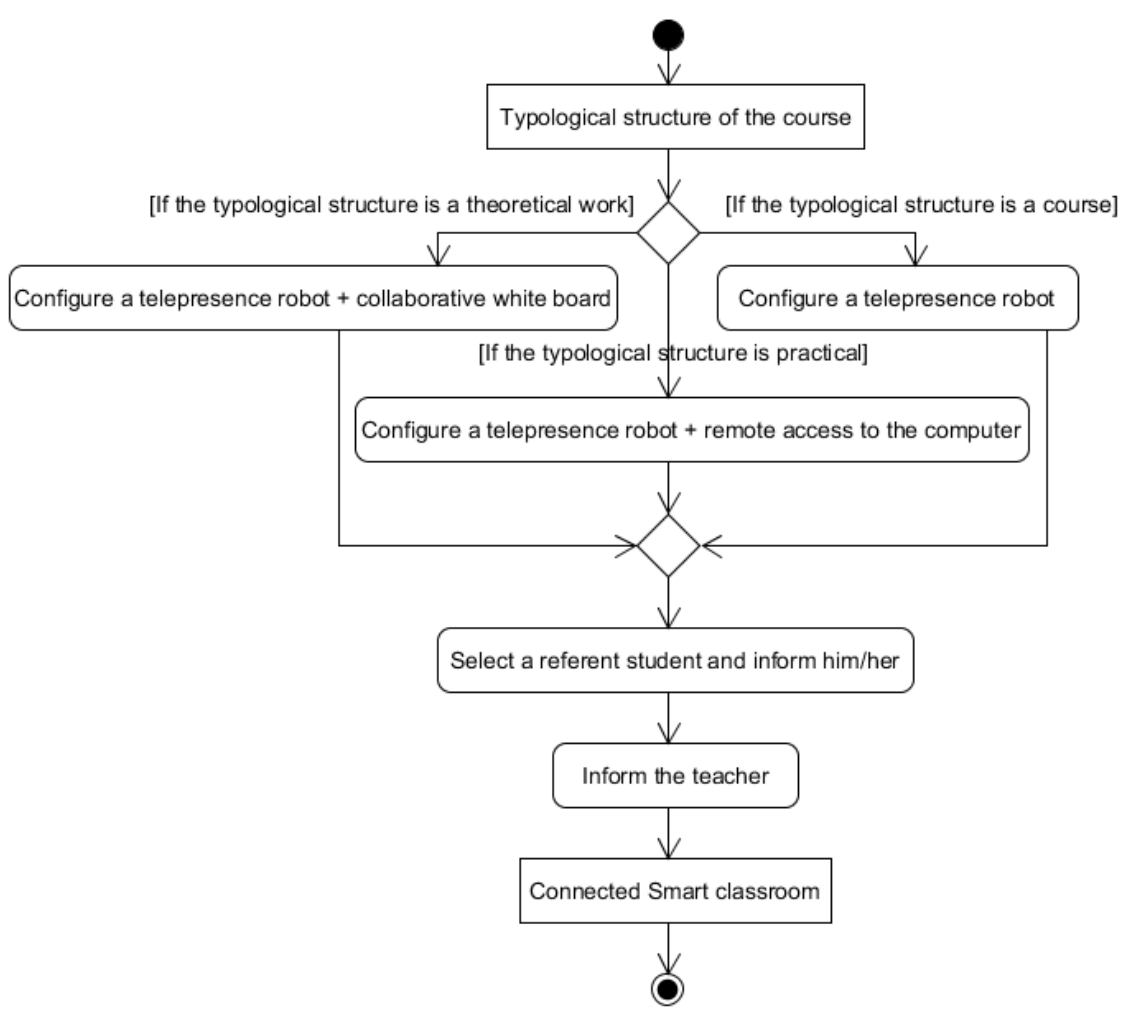

Fig. 3. Example of a classroom process

The followings are examples of sub-processes (a part of complete processes respectively) we propose, that can be generated by different ECPS according to some context parameters that may be required at a given time.

- Classroom process (Fig. 3): Aim: Configure the smart objects for a telepresent student according to the typological structure of the course. This also affects indirectly the interactions between people (students, teachers, etc.). If 


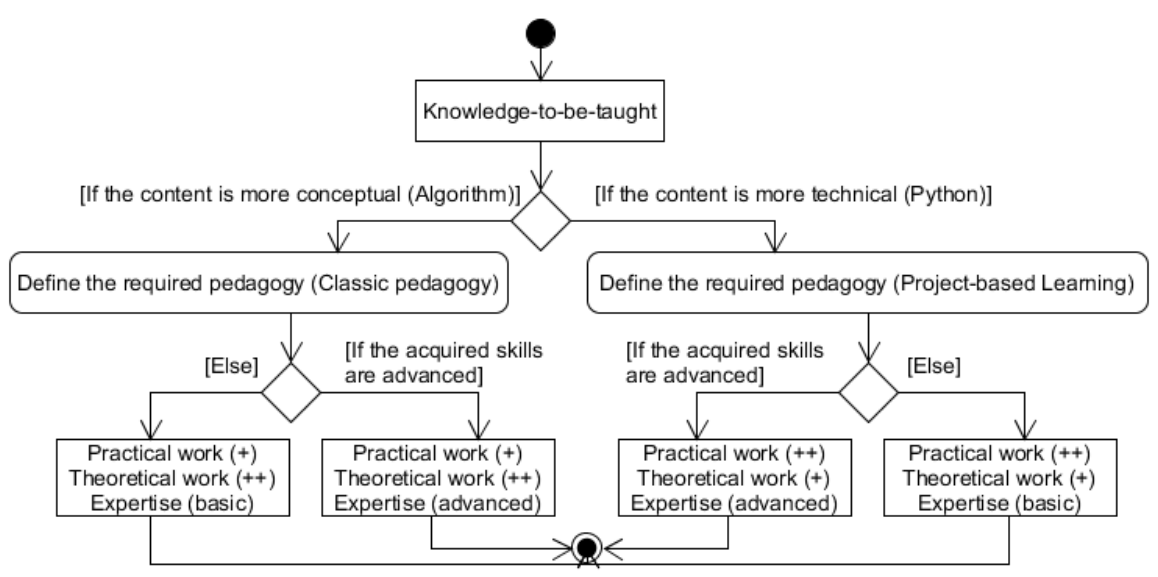

Fig. 4. Example of course processes

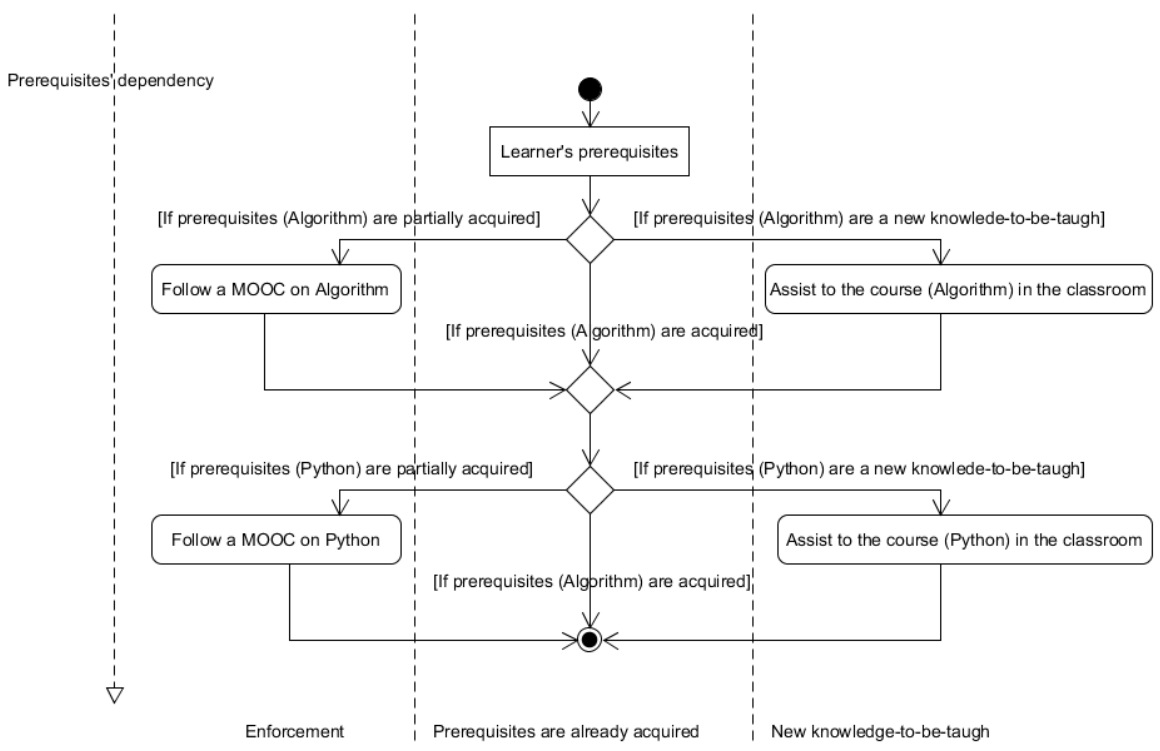

Fig. 5. Example of curriculum processes (upgrade level case)

we need to connect telepresent-students, according to the typological structure of the course, the correspondant ECPS will propose the right activity to do in order to configure the required objects for configuring the connection. Other sub-activities related to the selected action will also be defined consequently, like the designation of a referent student who will be in charge of the communication with the remote student. The teacher will be notified 
once the designation is done. This translates the kind of interactions between people that the ECPS reports according to the components of IoE.

- Course process (Fig. 4): Aim: Based on the knowledge-to-be-taught, the strategy of the course plan and learning objectives could change. In our example, if the knowledge-to-be-taught is more conceptual, we will design the course focusing more on theoretical aspects, elsewise, the focus will be on the practical works. The design of the knowledge-to-be-taugh will depend on the nature of the subject itself. In addition, according to the students' aquired skills, the design of the learning will differ for each student in order to personalise the required learning processes. For example, if a student has already acquired the basic skills, we can propose for him/her (or choose for him/her) to follow an an advanced course instead.

- Curriculum process (Fig. 5): Aim: Upgrade students' level: the adaptation of the curriculum according to collected data about student background. In the given example, if the learners' prerequisites are not acquired, we could propose them to follow a MOOC (Massive Open Online Course) or to assist to the course in the classroom if it is about a new subject to learn. They could have the possibility to not follow the course if it is proven that they have the required competences. Prerequisite dependency is a key parameter in every curriculum design, Algorithm before Python in this case. Hence, for the upgrade of students' level, one may find activities when it comes to enforcement, other activities for learning new knowledge and others when prerequisites are acquired.

Each type of process is continuously implemented, monitored and supervised by a specific ECPS which mobilizes different managed ressources according to an ongoing process. The given examples are not about complete processes but rather some samples of a set of activities that ECPS generate according to a given situation. The situation includes the different elements of the IoE (people, things, data, processes, learning/teaching context). In fact, the ECPS in charge of the curriculum processes will manage a set of ECPS running in a set of course processes, correspondingly. Similarly, the ECPS in charge of the course process will manage a set of ECPS running in a set of classroom processes. Finally, a classroom process run out by an ECPS could be specific for each student or a group of students to monitor and adapt the educational processes.

\section{Conclusion and Future Works}

Dealing with the Internet of Everything in the educational ecosystem is different from other domains. Various challenges about setting up a connected environment, coupled with optimising the educational experience as a whole package, drive us to rethink the different elements of IoE. The context factor is very interesting to study as it leads the way processes could get defined or adapted, something we have started doing in this paper. In this research work, we proposed a revised version of the Internet of Everything elements of the educational 
ecosystem and a key concept for University 4.0: ECPS. We are currently working on refining a generic reference architecture for university 4.0 through the bias of ECPS. This generic architecture will define the main global elements of such system and their interactions supported by bidirectional data flows (from physical to cyber and vice-versa). Then, we will define a model driven method to propose an Architecture Description for Educational Cyber Physical System. Different views will be provided by the bias of Domain Specific Language (DSL) to depict the different elements of an ECPS covering the IoE components.

\section{References}

1. Educause, https://er.educause.edu/articles/2016/6/the-internet-of-things-ridingthe-wave-in-higher-education, 2016 (accessed May 27, 2019).

2. Maryam Bagheri and Siavosh H Movahed. The effect of the internet of things (iot) on education business model. In 2016 12th International Conference on SignalImage Technology \& Internet-Based Systems (SITIS), pages 435-441. IEEE, 2016.

3. Barry Burd, Lecia Barker, Monica Divitini, Felix Armando Fermin Perez, Ingrid Russell, Bill Siever, and Liviana Tudor. Courses, content, and tools for internet of things in computer science education. In Proceedings of the 2017 ITiCSE Conference on Working Group Reports, pages 125-139. ACM, 2018.

4. Joe Cecil and Damon Chandler. Cyber physical systems and technologies for next generation e-learning activities. Cyber Physical Systems and Technologies for Next Generation e-Learning Activities, (10):1-11, 2014.

5. Monica Ciolacu, Paul Mugur Svasta, Waldemar Berg, and Heribert Popp. Education 4.0 for tall thin engineer in a data driven society. In Design and Technology in Electronic Packaging (SIITME), 2017 IEEE 23rd International Symposium for, pages 432-437. IEEE, 2017.

6. Autonomic Computing. An architectural blueprint for autonomic computing. IBM White Paper, 31:1-6, 2006.

7. Rafael de Amorim Silva and Rosana TV Braga. An acknowledged system of systems for educational internet of everything ecosystems. In Proceedings of the 12th European Conference on Software Architecture: Companion Proceedings, page 25. ACM, 2018.

8. Richard W Herling and Joanne Provo. Knowledge, competence, and expertise in organizations. Advances in Developing Human Resources, 2(1):1-7, 2000.

9. Mario Hermann, Tobias Pentek, and Boris Otto. Design principles for industrie 4.0 scenarios. In System Sciences (HICSS), 2016 49th Hawaii International Conference on, pages 3928-3937. IEEE, 2016.

10. Henning Kagermann, Wolfgang Wahlster, and Johannes Helbig. Final report of the industrie 4.0 working group. Federal Ministry of Education and Research, 82, 2013.

11. Jay Lee, Behrad Bagheri, and Hung-An Kao. A cyber-physical systems architecture for industry 4.0-based manufacturing systems. Manufacturing Letters, 3:18-23, 2015.

12. Filipe T Moreira, Andreia Magalhães, Fernando Ramos, and Mário Vairinhos. The power of the internet of things in education: an overview of current status and potential. In Conference on Smart Learning Ecosystems and Regional Development, pages 51-63. Springer, 2017.

13. Mohammad Reza Nami and Koen Bertels. A survey of autonomic computing systems. In Autonomic and Autonomous Systems, 200\%. ICAS0\%. Third International Conference on, pages 26-26. IEEE, 2007. 
14. Daniel Palma, Juan Agudo, Héctor Sánchez, and Miguel Macías. An internet of things example: Classrooms access control over near field communication. Sensors, 14(4):6998-7012, 2014.

15. Vichian Puncreobutr. Education 4.0: new challenge of learning. St. Theresa Journal of Humanities and Social Sciences, 2(2), 2016.

16. Dosheela Devi Ramlowat and Binod Kumar Pattanayak. Exploring the internet of things (iot) in education: A review. In Information Systems Design and Intelligent Applications, pages 245-255. Springer, 2019.

17. Samuel Mensah Sackey, Andre Bester, and Dennit Adams. Industry 4.0 learning factory didactic design parameters for industrial engineering education in south africa. South African Journal of Industrial Engineering, 28(1):114-124, 2017.

18. Divesh S Sharma. Accounting students' learning conceptions, approaches to learning, and the influence of the learning-teaching context on approaches to learning. Accounting Education, 6(2):125-146, 1997.

19. Temitope Takpor and Aderemi A Atayero. Integrating internet of things and ehealth solutions for students healthcare. In Proceedings of the World Congress on Engineering, volume 1. World Congress on Engineering, London, UK, 2015.

20. Yang Yuqiao and Yu Kanhua. Construction of distance education classroom in architecture specialty based on internet of things technology. International Journal of Emerging Technologies in Learning (iJET), 11(05):56-61, 2016. 